\title{
Procedimento Combinado Entre Operação de Revascularização do Miocárdio e Endarterectomia de Carótida. Análise dos Resultados
}

\author{
Combined Carotid Endarterectomy and Coronary Artery Bypass Grafting. \\ Analysis of the Results
}

Márcio Luís Lucas, Telmo Pedro Bonamigo, Elton Luiz Schmidt Weber, Fernando Antônio Lucchese

Santa Casa de Porto Alegre - Porto Alegre, RS

\section{Objetivo}

Avaliar os resultados da cirurgia combinada (endarterectomia carotídea e cirurgia de revascularização do miocárdio) em pacientes com doença carotídea e coronária concomitante.

\section{Métodos}

Os autores revisam 49 trabalhos que descrevem vários aspectos sobre a cirurgia combinada em um total de 4.788 pacientes, analisando eventos precoces no período peri-operatório.

\section{Resultados}

As taxas globais de acidente vascular cerebral (AVC), infarto agudo do miocárdio (IAM) e mortalidade foram, respectivamente, de 4,3\%; 2,2\%; e 4,2\%. Para os trabalhos mais recentes (entre 1990-2000), a ocorrência de AVC foi significativamente inferior àquela obtida por trabalhos publicados anteriormente (entre 1972-1989) (4,1\% x $10,2 \% ; p<0,05)$. Neste mesmo levantamento, os índices de AVC pós-operatórios foram maiores nos artigos que continham uma amostra pequena $(n<50)$ que naqueles trabalhos com uma população maior $(n>100)(7,2 \% x$ 3,9\%; $p<0,05$ ), denotando o impacto da experiência dos cirurgiões sobre os resultados pós-operatórios.

\section{Conclusão}

Em suma, acreditamos que a cirurgia combinada é um procedimento seguro e eficaz quando realizado por cirurgiões experientes e qualificados.

\section{Palavras-chave}

endarterectomia carotídea, revascularização coronariana, revisão

\section{ОвJective}

To evaluate the results of combined surgery (carotid endarterectomy and coronary artery bypass grafting) in patients with concomitant carotid and coronary artery disease.

\section{Methods}

The authors reviewed 49 different reports describing several aspects of the combined surgery in 4788 patients to analyze early events in the perioperative period.

\section{RESULTS}

Overall stroke, acute myocardial infarction (AMI), and mortality rates were $4.3 \%, 2.2 \%$, and $4.2 \%$, respectively. Most recent papers (1990 to 2000) show significant lower incidence of strokes compared to those published earlier (1972 to 1989) (4.1\% x 10.2\%; p < 0.05). In this same survey, the incidence of postoperative stroke was higher in the series with a small sample $(n<50)$ than in those with a larger population ( $n>$ 100) $(7.2 \% \times 3.9 \%$; $p<0.05)$, denoting the impact of surgeons' experience on postoperative results.

\section{Conclusion}

In sum, we believe that combined carotid endarterectomy and coronary artery bypass grafting is a safe and effective procedure when performed by experienced and qualified surgeons.

\section{KEYWORDS}

carotid endarterectomy, coronary artery bypass grafting, review 
As indicações para a cirurgia de revascularização do miocárdio (RM) e para a endarterectomia de carótida (EC) isoladas estão bem estabelecidas na literatura corrente ${ }^{1-4}$. Cerca de $8 \%$ a $14 \%$ dos pacientes que são submetidos a RM possuem estenose carotídea significativa (> 70\%), e quase metade dos pacientes submetidos a EC tem certo grau de coronariopatia ${ }^{5-7}$, sendo controverso o manejo cirúrgico desses doentes ${ }^{8-10}$.

Existem várias táticas e técnicas operatórias para o tratamento de pacientes portadores de doença coronariana obstrutiva e doença carotídea associadas, sendo as mais descritas na literatura a cirurgia combinada (os procedimentos são realizados sob a mesma anestesia), a cirurgia simultânea (sincrônica ou concomitante), em que há participação de duas equipes cirúrgicas ao mesmo tempo, e a cirurgia seqüencial, sendo realizada primeiro a EC ou a RM em um mesmo tempo anestésico para ambas; a cirurgia estagiada, em que a EC precede a RM; e, por fim, a cirurgia estagiada reversa, em que se opta por realizar a cirurgia cardíaca primeiro, postergandose a correção do defeito carotídeo por alguns dias, até recuperação da primeira cirurgia ${ }^{11}$.

Embora não esteja definida uma superioridade entre os resultados da cirurgia combinada e estagiada, grande parte dos cirurgiões vasculares e cardiovasculares propõe a realização do procedimento combinado 7 .

O objetivo da nossa revisão é abordar aspectos do perfil clínico desses pacientes, indicações cirúrgicas, táticas e técnicas operatórias, resultados e complicações no pósoperatório a respeito da cirurgia combinada em pacientes com doença aterosclerótica avançada.

\section{Métodos}

\section{Perfil clínico dos pacientes}

$\mathrm{Na}$ maioria das vezes, os pacientes portadores de doença aterosclerótica nos dois setores arteriais (carotídeo e coronariano) são indivíduos de idade avançada, portadores de fatores de risco para a doença aterosclerótica como hipertensão, tabagismo, dislipidemia e diabetes. A maioria é obesa, sedentária, com história familiar positiva para coronariopatia e história prévia de algum evento neurológico ou cardiovascular. Em suma, esses pacientes merecem atenção e cuidados redobrados no período perioperatório, pois são mais propensos a desenvolver complicações graves após a cirurgia, como acidente vascular cerebral (AVC), infarto agudo do miocárdio (IAM) e morte.

A média de idade dos pacientes é de 65 anos, sendo a prevalência no sexo masculino até três vezes maior que no feminino ${ }^{12,13}$. Alguns estudos têm demonstrado que pacientes com idade maior ou igual a 75 anos têm maior probabilidade de desenvolver AVC pós-operatório, quando comparado a pacientes com idade inferior a 65 anos $^{7}$.
A freqüência de cada fator de risco está representada

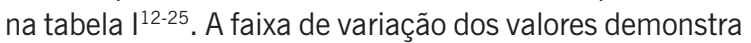
a heterogeneidade das amostras dos diversos estudos, e hipertensão e tabagismo foram os fatores mais freqüentes. Além disso, o grau de aterosclerose sistêmica pode ser evidenciado também pela freqüência da arteriopatia periférica nesses pacientes ${ }^{14,15}$.

Tabela I - Variação da freqüência de fatores

de risco para doença cardiovascular em pacientes

submetidos à cirurgia combinada (EC+RM) $)^{12-25}$

\begin{tabular}{lr} 
Hipertensão arterial sistêmica & $39 \%$ a $91 \%$ \\
\hline Tabagismo & $26 \%$ a $81 \%$ \\
\hline Diabete melito & $8 \%$ a $48 \%$ \\
\hline Obesidade & até $55 \%$ \\
\hline Dislipidemia & $35 \%$ a $60 \%$ \\
\hline Arteriopatia periférica & $24 \%$ a $58 \%$
\end{tabular}

A história cardiológica é um dado importante na avaliação pré-operatória dos pacientes a serem submetidos ao procedimento combinado. Os índices de infarto agudo do miocárdio (IAM) prévio à cirurgia podem variar de $41 \%$ a $67 \%{ }^{8,16}$. Cerca de $20 \%$ a $30 \%$ dos pacientes sofrem de insuficiência cardíaca congestiva ${ }^{16,17}$ e a freqüência de angina instável está entre $20 \%$ e $67 \%{ }^{7,9}$. A maioria dos pacientes anginosos (70\% a 75\%) encontra-se nas classes III e IV, segundo a NYHA (New York Heart Association) ${ }^{12,15,16}$. No estudo angiográfico préoperatório, cerca de $60 \%$ a $80 \%$ dos doentes possuem lesão de três vasos coronarianos e de tronco principal esquerdo ${ }^{10,18}$. A fração de ejeção menor que $50 \%$ pode estar presente em até $72 \%$ dos pacientes ${ }^{18}$.

$\mathrm{Na}$ avaliação neurológica, os pacientes podem apresentar sintomas prévios em até $70 \%$ dos $\operatorname{casos}^{16}$, sendo acidente isquêmico transitório (AIT), o acidente neurológico prolongado reversível (ANPR) e a amaurose fugaz presentes em $20 \%-60 \%$, até $16 \%$ e até $8 \%$, respectivamente ${ }^{8,9,19}$. Além disso, o acidente vascular cerebral permanente (AVC) foi previamente diagnosticado em cerca de $20 \%$ dos pacientes em algumas casuísticas ${ }^{16,18,26,27}$. 0 grau de lesão carotídea também foi outro fator bem estudado. Desse modo, a média de estenose, em alguns estudos, ultrapassou $85 \%{ }^{18,26}$. A lesão significativa bilateral chega a estar presente em $60 \%$ a $90 \%$ dos casos ${ }^{14,16}$. Lesão contralateral significativa chega a uma freqüência entre 20\% e 40\% ${ }^{7,12}$, enquanto oclusão contralateral total foi encontrada entre $10 \%$ e $48 \%$ dos pacientes operados ${ }^{8,18}$. No estudo de Trachiotis \& Pfister $^{18}$, a média de estenose nos pacientes com lesão bilateral foi de $86,2 \%$ no lado operado, contra $81,5 \%$ no lado oposto, enquanto para Mackey e cols. ${ }^{26}$ esses índices foram 88,8\% e 75,3\%, respectivamente, ressaltando o potencial de complicações pós-operatórias desses doentes. Assim, não raramente tratamos pacientes portadores de lesão carotídea bilateral, e o manejo desses enfermos deve ser bem estudado. A maioria dos autores é relutante em corrigir a lesão carotídea bilateral junto com a cirurgia cardíaca, sendo realizada, primeiramente, a endarterectomia carotídea no lado que contribui para o 
maior fluxo cerebral; isto é, o lado com estenose menor. No entanto, Dylewski e cols. ${ }^{14}$ reportaram sua experiência em 33 pacientes submetidos a RM e EC bilateral simultaneamente. Noventa e quatro por cento dos pacientes tinham lesão do lado direito, e 91\% do lado esquerdo. Não ocorreu AVC ou IAM nessa casuística, e mortalidade e complicações pulmonares estiveram presentes em $6 \%$ e $12 \%$ dos pacientes, respectivamente.

\section{Indicação e técnica cirúrgicas}

Alguns estudos demonstraram a ocorrência de lesão carotídea no pré-operatório de pacientes portadores de doença coronariana obstrutiva. Assim, programas de rastreamento em candidatos a RM podem identificar de $2,8 \%$ a $8,7 \%$ dos pacientes com lesão carotídea significativa $(\geq 75 \%)^{28-33}$.

A freqüência de cirurgias combinadas (RM + EC) entre as cirurgias de revascularização do miocárdio varia de $0,7 \%$ a $4,2 \%{ }^{17,18}$, sendo um procedimento que vem em expansão em alguns centros em razão do aumento da confiança na eficácia dessa abordagem, bem como uma maior incidência de idosos portadores de doença aterosclerótica avançada7.

A recomendação para a cirurgia combinada pressupõe a existência de angina instável, lesão significativa no tronco coronariano principal esquerdo, instabilidade hemodinâmica, lesão de múltiplos vasos em paciente com doença carotídea sintomática na presença de estenoses significativas ou lesão bilateral ${ }^{8}$. Em pacientes com lesão carotídea assintomática, a cirurgia não estaria indicada, pois o risco de AVC é similar ao risco cirúrgico ${ }^{11}$. Por sua vez, a cirurgia estagiada seria realizada em pacientes mais estáveis do ponto de vista hemodinâmico e com lesão de um ou dois vasos coronarianos ${ }^{9}$. No entanto, essa distinção de conduta não está bem definida. Alguns autores têm demonstrado sua experiência com a cirurgia combinada em pacientes muitas vezes estáveis e assintomáticos do ponto de vista neurológico. A escolha do procedimento (estagiado ou combinado) depende das condições do paciente, bem como da experiência dos cirurgiões em realizar um ou outro procedimento. Além disso, o ideal para o tratamento cirúrgico dos pacientes com ambas as lesões seria a forma eletiva; porém os índices de cirurgia de urgência ou de emergência podem ir de $33 \%$ a $72 \%$ dos $\operatorname{casos}^{7,14}$.

A maioria dos autores indica cirurgia de carótida quando há lesão significativa unilateral, sintomas neurológicos prévios ou presença de placa ateromatosa ulcerada. No entanto, o ponto de corte para estabelecer lesão significativa varia de $70 \%$ a $80 \%^{1,8,18}$. Outras indicações, como lesão bilateral $\geq 50 \%$, lesão unilateral $\geq 50 \%$ com o outro lado ocluído e presença de sopro carotídeo, também são levadas em conta por outros autores $^{9,13,15,16,18,19,26,34,35}$.

Em alguns estudos, a EC precedeu a RM na mesma anestesia sem o auxílio da circulação extracorpórea
$(\mathrm{CEC})^{7,9,12,17,26,36}$. No entanto, Khaitan e cols. ${ }^{13}$ demonstraram bons resultados com procedimento combinado e o auxílio da CEC. Esses autores observaram que o aumento no tempo de CEC determinou um incremento na morbidade e mortalidade e no tempo de internação em unidade de tratamento intensivo (UTI). De outra forma, o trabalho de Schwartz e cols. ${ }^{37}$ não mostrou influência significativa da CEC sobre as taxas de morbidade e mortalidade. Minami e cols. ${ }^{8}$ também analisaram o efeito da CEC sobre a cirurgia combinada em 340 pacientes, achando que tal abordagem poderia causar algum grau de neuroproteção, diminuindo as complicações pósoperatórias. A hipótese seria de que a hipotermia, causada pela CEC, diminuiria o aporte de oxigênio cerebral, bem como atenuaria a produção de metabólitos anóxicos cerebrais. Porém, os resultados desses autores podem ser comparados com os obtidos por outros pesquisadores que não utilizaram CEC durante a EC.

A decisão sobre para quais pacientes se deve indicar a cirurgia combinada, bem como quando realizá-la é assunto de ampla discussão. Alguns autores demonstraram que a RM sem o tratamento concomitante da lesão carotídea significativa pode ter índice de complicações neurológicas variando de $7 \%$ a $61 \%$ e $1,6 \%$ a $23 \%$ para lesões transitórias e permanentes, respectivamente ${ }^{38}$. Há um aumento no risco de AVC perioperatório de aproximadamente três vezes em pacientes com história de isquemia cerebrovascular prévia ${ }^{31}$. Quando realizado o procedimento combinado, esses índices caem em torno de $10 \%$ para as lesões neurológicas ${ }^{26,27,39-44}$. Outros fatores de risco importantes para a ocorrência de dano neurológico pós-RM são idade avançada e tempo prolongado de $\mathrm{CEC}^{45}$. Entretanto, a ocorrência de IAM pós-EC é amplamente variável. Em pacientes sem história de sintomatologia cardíaca, a incidência é tão baixa quanto $1 \%$, enquanto nos pacientes com sintomatologia essas taxas podem chegar de $7 \%$ a $17 \%{ }^{46}$.

Os fundamentos que embasam a indicação da cirurgia combinada repousam em três pontos principais: primeiro, a doença carotídea não-diagnosticada pode ser um agravante para o dano neurológico perioperatório em pacientes submetidos a RM; desse modo, a correção da lesão carotídea antes do estabelecimento da CEC reduziria esse risco. Segundo, o procedimento seqüencial, iniciandose pela EC, proporcionaria um aumento na morbidade e mortalidade cardiovascular, visto que as síndromes cardíacas isquêmicas são as principais complicações em pacientes sujeitos a correção da doença carotídea. Por último, ao propor o tratamento cirúrgico de apenas um setor arterial (carotídeo ou coronariano), tem-se o risco de se transformar um quadro clínico estável em instável ${ }^{45}$.

A literatura tem demonstrado que a presença de doença carotídea aumenta significativamente a probabilidade de ocorrência de AVC após a $\mathrm{RM}^{28,47,48}$. Assim, a EC deveria preceder a RM em caráter combinado ou estagiado, dependendo das condições do paciente, da disponibilidade das equipes cirúrgicas, bem como da 
experiência cirúrgica local. No intuito de elucidar qual seria a melhor abordagem para esses pacientes, Borger e cols. ${ }^{5}$ publicaram uma metanálise de dezesseis estudos confrontando os resultados das cirurgias combinada e estagiada. Essa metanálise verificou que o grupo combinado $(n=844)$ apresentou índices de AVC $(6 \% x$ $3,2 \%)$ e mortalidade $(4,75 \% \times 2,9 \%)$ significativamente superiores aos do grupo estagiado ( $\mathrm{n}=920$ ). Porém, a ocorrência de IAM no grupo combinado $(4,6 \%)$ não diferiu significativamente do grupo estagiado (5,1\%). Esses resultados devem ser devidos, provavelmente, à maior gravidade de doenças associadas aos grupos dos pacientes submetidos ao procedimento combinado nesses estudos.

No estudo de Coyle e cols. ${ }^{10}$, identificaram-se maiores índices de morbidade e mortalidade (AVC e morte) após cirurgia combinada quando comparada à estagiada. Deve ser lembrado que os pacientes sintomáticos, do ponto de vista neurológico, com maior grau de estenose carotídea, hipertensos, tabagistas, anginosos e com lesão em três vasos, foram mais prevalentes no grupo combinado, quando comparado ao grupo estagiado. Esses dados comprovam a maior gravidade dos pacientes submetidos ao procedimento combinado na maioria dos ensaios. Além disso, é preciso realizar um estudo totalmente randomizado para determinar se existem ou não diferenças entre os resultados das duas cirurgias. Tal estudo necessitaria da participação de várias instituições para adquirir amostra populacional de tamanho confiável.

Segundo os cálculos de Borger e cols. ${ }^{5}$, para uma incidência de desfecho final (por exemplo, AVC) de $7,5 \%$, necessitaríamos uma amostra de 1.500 pacientes por grupo para detectar uma redução de risco relativo de 33\% com um nível alfa de 0,05. De forma similar, Barnes e cols. ${ }^{49}$ revisaram um total de 1.483 pacientes com doença carotídea assintomática subdivididos nos dois procedimentos. Esse estudo obteve menores taxas de IAM $(3,8 \%$ x $11,8 \%)$ e mortalidade $(4,7 \% \times 11,1 \%)$ no grupo combinado; entretanto, a ocorrência de AVC entre o grupo combinado $(2,8 \%)$ não mostrou diferença estatística significativa ao grupo estagiado $(3,1 \%)$, sendo esses resultados divergentes dos obtidos pela metanálise de Borger e cols. ${ }^{5}$.

Com relação ao procedimento cirúrgico em si, alguns pontos podem ser discutidos pela variabilidade de técnicas utilizadas nos estudos. Um grande número de autores utiliza monitorização eletroencefalográfica transoperatória para evidenciar possíveis alterações secundárias à hipoperfusão cerebral, utilizando shunt a partir do momento em que tais alterações apareçam ${ }^{8,12,16,36}$. Além disso, hipotermia (temperatura entre $27-30^{\circ} \mathrm{C}$ ) e hemodiluição (hematócrito entre 20\%-25\%) parecem exercer algum efeito neuroprotetor durante a CEC. No entanto, a hipotermia, por vezes, pode mascarar as possíveis alterações eletroencefalográficas isquêmicas, retardando o uso criterioso de shunt interno no transoperatório $^{8}$. O uso desse dispositivo pode ser uma decisão do cirurgião ${ }^{7}$, ou embasada pela presença de oclusão contralateral ${ }^{15}$. Alguns autores abrem mão do uso da monitorização eletroencefalográfica e não usam shunt rotineiramente ${ }^{13}$; outros o utilizam sempre ${ }^{17,18}$. Dylewski e cols. ${ }^{14}$, ao contrário da técnica convencional de endarterectomia carotídea, empregaram a técnica de eversão bilateral em 33 pacientes, obtendo índices de AVC e IAM nulos e mortalidade de 6,1\%.

O emprego de remendo não é uma unanimidade, sendo utilizado rotineiramente por alguns autores ${ }^{8,15}$, e somente quando o diâmetro da carótida interna for menor que 4 $\mathrm{mm}$, por outros ${ }^{15,17}$. Arteriorrafia primária sem uso de remendo é relatada como procedimento de escolha em outros trabalhos $7,9,13$.

Fatores que determinam a duração da cirurgia como tempo de CEC, tempo de clampeamento e número de pontes por paciente também podem interferir nos resultados. O tempo de CEC é um importante preditor de risco para complicações pós-operatórias, variando entre 72 a 129 minutos $^{13,18}$. 0 tempo de pinçamento aórtico situa-se entre 32 e 44 minutos $^{10,14}$, e o número de pontes pode variar entre 2,9 e 4, por paciente ${ }^{8,36}$.

\section{Resultados}

Alguns trabalhos avaliaram os resultados após a cirurgia combinada mediante tempo de hospitalização, internação em UTI, ocorrência de complicações pulmonares, infecciosas ou renais, bem como sobrevida e ausência de eventos neurocardiovasculares a longo prazo. No entanto, os dados mais discutidos e apresentados foram os índices de AVC, IAM e mortalidade pós-operatórios.

Compilando os dados de 49 trabalhos com mais de quinze pacientes cada, em um total de 4.788 pacientes, que relataram a experiência de diversos centros na realização da cirurgia combinada, obtivemos índices de AVC entre 0 e 13,3\%, IAM de 0 e 10,4\%, e mortalidade de 0 e 11,5\%. A ocorrência global de AVC, IAM e mortalidade foi $4,3 \%, 2,2 \%$ e $4,2 \%$, respectivamente. Recentemente, um estudo conduzido por Brown e cols. ${ }^{50}$, envolvendo dez estados norte-americanos, analisou os resultados de 226 procedimentos combinados (RM + EC) em pacientes com idade média de 72,2 anos. As taxas de AVC (12\%) e mortalidade $(6,7 \%)$ foram superiores à maioria dos trabalhos por nós analisados, embora esses valores ainda se encontrem nas variações expostas anteriormente. Com esses resultados, Brown e cols. ${ }^{50}$ salientam a ponderação em indicar o procedimento combinado, pois a maioria dos pacientes analisados por esses autores era assintomática do ponto de vista de doença cerebrovascular, e que os sintomas da grande parte dos AVCs no pós-operatório não estavam relacionados com o território cerebral correspondente ao lado operado ${ }^{50}$.

Para verificar a influência da data de publicação do trabalho sobre os resultados de morbidade e mortalidade, distribuímos os artigos em dois grupos: o "antigo", 
constituído por 27 publicações no período entre 1972 e 1989; e o "recente", formado por 23 trabalhos publicados entre 1990 e 2000 . As médias de idade de cada grupo foram 62,2 e 68,6 anos, respectivamente. Os índices de AVC e IAM foram $10,2 \%$ e $4,1 \%$; e $2,5 \%$ e $2,1 \%$, respectivamente. Para a mortalidade, as taxas ficaram em 5,4\% para o grupo "antigo" e 3,8\% para o "recente". Através do teste do qui-quadrado $\left(\mathrm{x}^{2}\right)$, considerando-se significância estatística $p<0,05$, constatamos que a ocorrência de AVC pós-operatório foi significativamente superior no grupo "antigo", mesmo sendo as idades dos pacientes desse grupo significativamente inferiores ao do grupo "recente" $(p<0,05)$. Não houve diferença entre os grupos com relação à mortalidade e ocorrência de IAM pós-operatório (tab. II).

Em uma revisão sistemática recente, Naylor e cols. ${ }^{51}$ analisaram os resultados da cirurgia simultânea de 94 trabalhos, em um total de 7.863 procedimentos. Esses autores observaram uma taxa de AVC, IAM e morte de $4,6 \%$; $3,6 \%$; e $4,6 \%$, respectivamente. Por meio da análise dos resultados conforme a data da publicação (1972-1992 x 1993-2002), observou-se que houve uma diminuição na taxa de morte $(5,2 \%$ x 4,4\%); AVC (6,5\% $\times 3,3 \%)$ e IAM (4,3\% x 3,4\%), sendo a taxa combinada de $\mathrm{AVC} /$ morte/IAM significativamente mais elevada no primeiro grupo $(13,8 \% \times 8,6 \% ; p=0,03)^{51}$.

A maioria absoluta das referências dessa revisão provém da língua inglesa. No Brasil, há poucos relatos sobre 0 assunto. Fichino e cols. ${ }^{52}$ relataram seus resultados após a cirurgia combinada em trinta pacientes operados. Não se observou nenhum caso de IAM perioperatório, e dois pacientes sofreram AIT (6,6\%) e outros dois evoluíram para o óbito $(6,6 \%)$, sendo as causas AVC e baixo débito cardíaco. No grupo de 46 pacientes operados por Souza e cols. ${ }^{53}$, entre janeiro de 1979 e novembro de 1994, a incidência de AVC e IAM foi nula, ocorrendo quatro mortes $(8,6 \%)$, sendo as causas: baixo débito cardíaco/insuficiência respiratória $(n=1)$, ruptura de aorta descendente $(n=1)$, óbito durante cirurgia para revascularização de membros inferiores ( $n=1)$, e insuficiência de múltiplos órgãos.

Tais resultados talvez ilustrem o aprimoramento das técnicas operatórias e anestésicas, bem como treinamento qualificado de cirurgiões vasculares e cardíacos, anestesistas e intensivas no manejo e abordagem dos pacientes submetidos a cirurgia combinada. No entanto, nossa análise não é isenta de viés na seleção dos pacientes, podendo haver diferenças nas características demográficas, clínicas e angiográficas dos doentes que compuseram cada grupo. Mesmo assim, esses resultados gerais oferecem certa credibilidade na realização da cirurgia combinada, que apresenta índices de morbidade e mortalidade aceitáveis para uma cirurgia de grande porte e, a cada dia, vem sendo mais realizada pelos cirurgiões cardiovasculares.

De forma similar, Rizzo e cols. ${ }^{16}$ analisaram dezesseis trabalhos com mais de cinqüenta pacientes, publicados entre 1979 e 1992, compreendendo 1.815 pacientes, e encontraram taxas globais de AVC, IAM e mortalidade de $5,6 \%, 3 \%$ e $4,8 \%$, respectivamente, sendo valores similares aos nossos, embora apenas um trabalho contido nessa revisão não tenha sido computado por nós.

O tempo médio de permanência hospitalar após a cirurgia combinada pode variar entre 10,3 e dezesseis dias $^{26,36}$, e o tempo de internação na UTI ficar entre 3,6 e quatro dias ${ }^{13,18}$. Complicações respiratórias ocorreram em até $12 \%$ dos pacientes ${ }^{14}$; insuficiência renal aguda em $6 \%^{14,36}$; sangramento necessitando de reintervenção cirúrgica em $5 \%{ }^{36}$; infecção da ferida operatória e hematoma em $4 \%^{10,36}$; síndrome de baixo débito cardíaco entre $0,6 \%$ e $4 \% \%^{8,36}$; arritmias em até $33 \%^{26}$; e fibrilação atrial de 0 a $34 \%{ }^{15}$.

Outro dado interessante foi a freqüência de AVC contralateral, que pode alcançar até $50 \%$ dos casos de AVC no pós-operatório, salientando a possibilidade de haver outras causas para essas complicações neurológicas, como embolia de origem cardiogênica ou aórtica, hemorragia intracraniana e diminuição da perfusão cerebral, como acontece nos momentos de hipotensão arterial| ${ }^{27}$.

A taxa de pacientes livres de AVC varia entre $67 \%$ e $96,4 \%^{7,26}$, e a taxa dos pacientes livres de IAM, entre $60 \%$ e $84 \% 7,16$. Em alguns trabalhos, a incidência de AVC após dez anos de cirurgia é de $11,8 \%$ no lado oposto e $9,3 \%$ no ipsilateral ${ }^{8}$. A sobrevida em dois, três, cinco e dez anos pode chegar a $94,2 \%, 81 \%, 78,9 \%$ e $56,1 \%$, respectivamente ${ }^{10,12}$. A mortalidade tardia nesses pacientes é atribuída principalmente a eventos cardíacos (62\%), sendo disfunção de ventrículo esquerdo, reoperação, idade avançada e história de IAM os fatores mais freqüentes ${ }^{16}$.

\begin{tabular}{|c|c|c|c|c|c|c|}
\hline Grupos & Trabalhos(n) & Pacientes(n) & Idade (anos) ${ }^{\mathrm{a}}$ & $\operatorname{AVC}(\%)$ & IAM(\%) & Mortes(\%) \\
\hline “antigo" (1972-1989) & 26 & 1801 & 62,2 & 10,2 & 2,5 & 5,4 \\
\hline "recente" (1990-2000) & 23 & 2987 & $68,6^{*}$ & $4,1^{*}$ & 2,1 & 3,8 \\
\hline $\mathrm{p}$ & & & 0,02 & 0,02 & 0,421 & 0,338 \\
\hline Total & 49 & 4788 & 65,2 & 4,3 & 2,2 & 4,2 \\
\hline
\end{tabular}


Para verificarmos a influência da experiência dos autores em realizar a cirurgia combinada sobre os resultados pós-operatórios, subdividimos em três grupos os resultados dos 49 trabalhos, conforme o número de pacientes por artigo estudado, sendo o primeiro grupo composto por artigos com menos de cinqüenta pacientes; o segundo, com um número de doentes entre cinqüenta e 99; e o último grupo com trabalhos contendo cem ou mais pacientes cada (tab. III). Por esse ângulo, à medida que aumenta a amostra populacional dos estudos, a ocorrência da complicação mais temível após a cirurgia combinada - o AVC - vai diminuindo significativamente de 7,2\% no grupo com menos de cinqüenta pacientes, para 3,9\% naqueles trabalhos com cem pacientes ou mais cada ( $p$ $<0,05)$. Tal diferença não foi observada pela análise das ocorrências de IAM e mortes no pós-operatório.

Esses resultados são similares àqueles apresentados por Takach e cols. ${ }^{54}$, que obtiveram índices de AVC significativamente superiores no grupo operado entre 1975-1985, quando comparado ao grupo de 19861996 (5,4\% x 1,9\%; p < 0,05). Para esses mesmos autores, a ocorrência de IAM e mortes no pós-operatório não demonstrou diferença significativa entre os grupos. No entanto, não distingüimos em nossa análise a densidade de pacientes operados pelo tempo de estudo. Assim, um estudo compreendendo 49 pacientes tratados cirurgicamente em um ano poderia pertencer ao mesmo grupo de um estudo que analisou 150 casos operados em três anos, sendo equivalentes pela densidade de casos operados por ano de estudo, que nesse exemplo seria de aproximadamente cinqüenta pacientes/ano.

A tabela IV demonstra resultados com relação a dados demográficos, clínicos e desfechos pós-operatórios de estudos recentes com cem ou mais pacientes cada. Os resultados dizem respeito a 1.663 pacientes operados em centros diferentes, cujas características demográficas, clínicas e angiográficas são apresentadas. Podemos observar que a ocorrência de AVC, IAM e mortes no pós-operatório imediato ( $\leq$ trinta dias) são de $4,6 \%$, $5,0 \%$, e $1,7 \%$, respectivamente, sendo semelhantes àquelas obtidas pela análise mais ampla $(n=4.788)$ anteriormente apresentada. Nota-se também que há uma tendência de os cirurgiões vasculares e cardiovasculares operarem, mais freqüentemente, pacientes mais idosos, visto que a média de idade para Rizzo e cols. ${ }^{16}$ e Khaitan e cols. ${ }^{13}$ variou de 65 a 69,2 anos, respectivamente.

Como foi dito anteriormente, sintomatologia neurológica, doença carotídea bilateral, envolvimento

\begin{tabular}{|c|c|c|c|c|c|}
\hline Pacientes/grupo & Trabalhos(n) & Total de pctes. & $\operatorname{AVC}(\%)$ & IAM(\%) & Mortes(\%) \\
\hline $\mathrm{n}<50$ & 13 & 362 & $26(7,2)^{\#}$ & $7(2,1)^{*}$ & $15(4,1)$ \\
\hline $50 \leq n<100$ & 17 & 1189 & $53(4,4)$ & $15(1,7)^{* *}$ & $63(5,3)$ \\
\hline$n>100$ & 19 & 3237 & $128(3,9)$ & $51(2,4)^{* * *}$ & $124(3,8)$ \\
\hline Total & 49 & 4788 & $207(4,3)$ & $73(2,2)$ & $202(4,2)$ \\
\hline
\end{tabular}

Tabela IV - Resultados da cirurgia combinada de trabalhos recentes com 100 ou mais pacientes cada

\begin{tabular}{|c|c|c|c|c|c|c|c|c|c|c|}
\hline Autor (referência) & Ano & $\begin{array}{l}\text { Pacientes } \\
\text { (n) }\end{array}$ & $\begin{array}{l}\text { Idade média } \\
\quad \text { (anos) }\end{array}$ & $\begin{array}{c}\text { Sintomas } \\
\text { neurológicos } \\
\text { (\%) }\end{array}$ & $\begin{array}{c}\text { Doença } \\
\text { bilateral } \\
(\%)\end{array}$ & $\begin{array}{c}\text { Doença tronco } \\
\text { esquerdo } \\
\text { (\%) }\end{array}$ & $\begin{array}{c}\text { Lesão } 3 \\
\text { vasos } \\
(\%)\end{array}$ & $\begin{array}{l}\text { AVC (\%) } \\
\text { perman. }\end{array}$ & IAM (\%) & Mortes (\%) \\
\hline Rizzo et al. ${ }^{16}$ & 1992 & 127 & 65 & $86(68)$ & $75(59)$ & $48(37)$ & $?$ & $7(5,5)$ & $6(4,7)$ & $7(5,5)$ \\
\hline Vermeulen et al. ${ }^{12}$ & 1992 & 230 & 65,2 & $108(47)$ & $92(40)$ & $67(29)$ & $185(80)$ & $13(5,6)$ & $4(1,8)$ & $8(3,5)$ \\
\hline Chang et al. ${ }^{19}$ & 1994 & 189 & 66 & $51(25)$ & $?$ & $?$ & $?$ & $2(1,0)$ & $?$ & $4(2,0)$ \\
\hline Coyle et al. ${ }^{10}$ & 1995 & 110 & 66,5 & $59(53,6)$ & $?$ & & $96(87,3)^{*}$ & $12(10,9)$ & $1(0,9)$ & $8(7,3)$ \\
\hline Akins et al. ${ }^{7}$ & 1995 & 200 & 67 & $85(42)$ & $44(22)$ & $86(43)$ & $130(65)$ & $8(4)$ & $5(2,5)$ & $7(3,5)$ \\
\hline Mackey et al. ${ }^{26}$ & 1996 & 100 & 68 & $57(57)$ & $61(61)$ & $?$ & $?$ & $9(9)$ & $6(6)$ & $8(8)$ \\
\hline Daily et al. ${ }^{36}$ & 1996 & 100 & 68 & $21(21)$ & $51(51)$ & $20(20)$ & $76(76)$ & $0(0)$ & $1(1)$ & $4(4)$ \\
\hline Takach et al..$^{54}$ & 1997 & 255 & 65,6 & $93(36,5)$ & $83(32,6)$ & $75(29,4)$ & $?$ & $10(3,9)$ & $12(4,7)$ & $10(3,9)$ \\
\hline Khaitan et al. ${ }^{13}$ & 2000 & 112 & 69,2 & $21(19)$ & $25(22,3)$ & $25(21)$ & $93(77)$ & $7(5,8)$ & $?$ & $7(5,8)$ \\
\hline Minami et al. ${ }^{8}$ & 2000 & 340 & 65,3 & $156(45,6)$ & $151(44,4)$ & $33(9,7)$ & $?$ & $11(3,2)$ & $2(0,6)$ & $9(2,6)$ \\
\hline TOTAL & & 1663 & & $737(44,3)$ & $582(42,6)$ & $354(28)$ & $484(75,4)$ & $77(4,6)$ & $83(5,0)$ & $72(4,3)$ \\
\hline Variação & & $100-340$ & $65-69,2$ & $19-68 \%$ & $22-61 \%$ & $9,7-43 \%$ & $65-80 \%$ & $0-10,9 \%$ & $0,6-6 \%$ & $2-6 \%$ \\
\hline
\end{tabular}


do tronco coronariano principal esquerdo, bem como lesão de múltiplos vasos denotam uma maior gravidade para os pacientes e, conforme a tabela IV, apresentam uma variabilidade de freqüência considerável. A média global de ocorrência para essas características foi de 44,3\%, $42,6 \%, 28 \%$, e $89 \%$, respectivamente, significando que quase metade dos pacientes operados tem sintomas neurológicos prévios e doença carotídea bilateral, enquanto em média um terço da amostra possui lesão de tronco principal esquerdo e grande parte dos pacientes tem lesão de múltiplos vasos.

\section{INFERÊNCIAS}

A ocorrência de doença carotídea e coronariana concomitantes é um achado freqüente em pacientes com doença aterosclerótica avançada por inúmeros fatores de risco cardiovasculares, como hipertensão, tabagismo, dislipidemia, obesidade e diabetes. 0 rastreamento de doença carotídea em pacientes com indicação de RM é um importante instrumento para a programação cirúrgica desses pacientes, visto que em vários trabalhos tem-se demonstrado que a doença carotídea aumenta o risco de complicações neurológicas após a RM, e que, se realizada a EC antes da RM, pode haver um impacto significativo nos resultados pós-operatórios a longo prazo. Entre as diferentes abordagens para o manejo cirúrgico da doença associada, a cirurgia combinada (simultânea) tem se demonstrado um procedimento seguro e eficaz, com índices de morbidade e mortalidade aceitáveis. 0 procedimento combinado possibilita apenas uma internação, com único procedimento anestésico, tratando duas doenças de morbidade e mortalidade importantes, diminuindo os riscos da doença que teria o tratamento postergado, bem como reduzindo os custos hospitalares.

Embora o número de cirurgias combinadas venha aumentando a cada ano, alguns estudos como o de Borger e cols. ${ }^{5}$ demonstram que os melhores resultados são obtidos com o procedimento estagiado; porém, não devemos nos esquecer de que os pacientes de maior risco e gravidade podem ser mais prevalentes no grupo combinado, e que nenhum ensaio totalmente randomizado foi realizado para esclarecer qual é o melhor procedimento.

O manejo dos pacientes com doença associada deve ser individualizado e bem ponderado; no entanto, algumas situações clínicas merecem destaque: sopro carotídeo assintomático: a indicação de RM parece ser o procedimento de eleição; porém, quando a estenose carotídea for crítica (90\%), estaria indicada a cirurgia combinada; AVC prévio: na presença de lesão carotídea importante (80\%) e angina estável, indica-se a cirurgia seqüencial. No entanto, na presença de angina instável, lesão de múltiplas coronárias ou lesão no tronco coronariano esquerdo, a cirurgia combinada poderá ser a melhor escolha; AIT prévio: na presença de lesão carotídea crítica, sugere-se a cirurgia combinada; caso contrário, o primeiro procedimento seria a RM; cirurgia carotídea prévia (reestenose da artéria carótida): se a lesão for maior que $50 \%$, a cirurgia seqüencial seria a escolha ${ }^{45}$.

De modo geral, a indicação clássica para a realização de cirurgia combinada seria em pacientes com angina instável, lesão em múltiplos vasos coronarianos, associada a lesão carotídea significativa (sintomática). Apesar de existirem algumas condutas técnicas controversas, como realização de endarterectomia carotídea sob circulação extracorpórea, utilização ou não de remendos e derivações, emprego de bombas de perfusão cardíaca, bem como outros artifícios para tentar diminuir o dano e as complicações pós-operatórias, consideramos aceitável um índice de morbidade e mortalidade total (ocorrência de acidentes vasculares cerebrais, infartos agudos do miocárdio e mortes) próximo a $10 \%-12 \%$, visto que, pela nossa análise, a ocorrência de AVC, IAM e mortalidade pós-operatórios gira em torno de 4,3\%, 2,2\% e 4,2\%, respectivamente.

Outro dado interessante é que a ocorrência de acidentes vasculares cerebrais após a cirurgia de revascularização miocárdica isolada pode chegar a $2 \%-3 \%$; e, após a endarterectomia carotídea isolada, até $2 \%-3 \%$; logo, a morbidade para esse desfecho nas cirurgias, se realizadas isoladamente, ficaria entre 4\% e 6\%, níveis bem próximos àqueles obtidos em nossa análise pelo procedimento combinado (4,3\%).

Em suma, embora pareça eficaz e segura, a cirurgia combinada para os pacientes com doença aterosclerótica sintomática nos setores carotídeo e coronariano deve ser bem indicada e realizada por cirurgiões, anestesiologistas e clínicos bem treinados, dedicados e capazes, amparados por uma equipe paramédica qualificada, na tentativa de sempre obter os melhores resultados para os pacientes.

\section{REFERÊNCIAS}

1. North American Symptomatic Carotid Endarterectomy Trial Collaborators. Beneficial effect of carotid endarterectomy in symptomatic patients with high-grade carotid stenosis. N Engl J Med 1991; 325: 445 .

2. Moore, WS, Barnett HJM, Beebe HG et al. Guidelines for carotid endarterectomy. A multidisciplinary consensus statement from ad hoc committee, American Heart Association. Stroke 1995; 26: 188-201.

3. European Carotid Surgery Trialists Collaborative Group. MRC European Carotid Surgery Trial: Interim results for syptomatic patients with severe (70-99\%) or with mild (0-29\%) carotid stenosis. Lancet 1991; 337: 1235 .

4. Yusuf S, Zucker D, Peduzzi P et al. Effect of coronary artery bypass graft on survival: overview of 10-year results from randomised trials by the Coronary Artery Bypass Surgery Trialists Collaboration. Lancet 1994; 344: 563-70.

5. Borger MA, Fremes SE, Weisel RD et al. Coronary bypass and carotid endarterectomy: does a combined approach increase risk? A metaanalysis. Ann Thorac Surg 1999; 68: 14-21.

6. Akins $\mathrm{CW}$. Combined carotid endarterectomy and coronary revascularization operation. Ann Thorac Surg 1998; 66: 1483-4.

7. Akins CW, Moncure AC, Daggett WM et al. Safety and efficacy of concomitant carotid and coronary operations. Ann Thorac Surg 1995; 60: 311-8. 
8. Minami K, Fukahara K, Boethig D et al. Long-term results of simultaneous carotid endarterectomy and myocardial revascularization with cardiopulmonary bypass used for both procedures. J Thorac Cardiovasc Surg 2000; 119: 764-73

9. Jahangiri M, Rees GM, Edmondson SJ et al. A surgical approach to coexistent coronary and carotid artery disease. Heart 1997; 77: 164-7.

10. Coyle KA, Gray BC, Smith RB et al. Morbidity and mortality associated with carotid endarterectomy: effect of adjunctive coronary revascularization. Ann Vasc Surg 1995; 9: 21-7.

11. Newmann DC, Hicks RG. Combined carotid and coronary artery surgery: a review of the literature. Ann Thorac Surg 1988; 45: 574-81.

12. Vermeulen FEE, Hamerlijnck RPHM, Defauw JJAM et al. Synchronous operation for ischemic cardiac and cerebrovascular disease: early results and long-term follow-up. Ann Thorac Surg 1992; 53: 381-90.

13. Khaitan L, Sutter FP, Goldman SM et al. Simultaneous carotid endarterectomy and coronary revascularization. Ann Thorac Surg 2000; 69: 421-4.

14. Dylewski M, Canver CC, Chanda J et al. Coronary artery bypass combined with bilateral carotid endarterectomy. Ann Thorac Surg 2001; 71: 777-82

15. Brow TD, Kakkar VV, Pepper JR et al. Toward a rational management of concomitant carotid and coronary artery disease. J Cardiovasc Surg 1999; 40: 837-44.

16. Rizzo RJ, Whittemore AD, Couper GS et al. Combined carotid and coronary revascularization: the preferred approach to the severe vasculopath. Ann Thorac Surg 1992; 54: 1099-109.

17. Bilfinger TV, Reda H, Giron F et al. Coronary and carotid operations under prospective standardized conditions: incidence and outcome. Ann Thorac Surg 2000; 69: 1792-8.

18. Trachiotis GD, Pfister AJ. Management strategy for simultaneous carotid endarterectomy and coronary revascularization. Ann Thorac Surg 1997; 64: 1013-8.

19. Chang BB, Darling RC, Shah DM et al. Carotid endarterectomy can be safely performed with acceptable mortality and morbidity in patients requiring coronary artery bypass grafts. Am J Surg 1994; 168: 94-6.

20. Snider F, Rossi M, Manni R et al. Combined surgery for cardiac and carotid disease: managment and results of a rational approach. Eur J Vasc Endovasc Surg 2000; 20: 523-7.

21. Char D, Cuadra S, Ricotta J et al. Combined coronary artery bypass and carotid endarterctomy: long term results. Cardiovasc Surg 2002; 10: $111-5$

22. Bernhard VM, Johnson WD, Peterson JJ. Carotid artery stenosis: association with surgery for coronary artery disease. Arc Surg 1972; 105: $837-40$

23. Ennix Jr CL, Lawrie GM, Morris Jr GC et al. Improved results of carotid endarterctomy in patients with symptomatic coronary disease: an analysis of 1546 consecutive carotid operations. Stroke 1979; 10: 122-5.

24. Rice PL, Pifarre R, Sullivan HJ, Montoya A, Bakhos M. Experience with simultaneous myocardial revascularisation and carotid endaterectomy. J Thor Cardiovasc Surg 1980; 79: 922-5.

25. Craver JM, Murphy DA, Jones EL et al. Concomittant carotid and coronary artery reconstruction. Ann Surg 1982; 195: 712-20.

26. Mackey WC, Khabbaz K, Bojar R et al. Simultaneous carotid endarterectomy and coronary bypass: perioperative risk and long-term survival. J Vasc Surg 1996; 24: 58-64.

27. Giangola G, Migaly J, Riles TS et al. Perioperative morbidity and mortality in combined versus staged approaches to carotid and coronary revascularization. Ann Vasc Surg 1996; 10: 138-42.

28. Schwartz LB, Bridgman AH, Kieffer RW et al. Asymptomatic carotid artery stenosis and stroke in patients undergoing cardiopulmonary bypass. J Vasc Surg 1995; 21: 146-53.

29. Berens ES, Kouchoukos NT, Murphy SF et al. Prospective carotid surgery screening in elderly patientes undergoing cardiac surgery. J Vasc Surg 1992; 15: 313-23.

30. Faggioli GL, Curl GR, Ricotta JJ. The role of carotid screening before coronary artery bypass. J Vasc Surg 1990; 12: 724-31.

31. Hertzer NR, Loop FD, Beven EG et al. Surgical staging for simultaneous coronary and carotid disease: a study including prospective randomization. J Vasc Surg 1989; 9: 455-63.

32. Minami K, Sagoo KS, Breymann T et al. Operative strategy in combined coronary and carotid artery disease. J Thorasc Cardiovasc Surg 1988; 95: 303:309.

33. Berkoff HA, Turnipseed WD. Patient selection and results of simultaneous coronary and carotid artery procedures. Ann Thor Surg 1984; 38: 172-5

34. Brener BJ, Brief DK, Alpert J, Goldekranz RJ, Parsonnet V. The risck of stroke in patients with assymptomatic carotid stenosis undergoing cardiac surgery: a follow-up study. J Vasc Surg 1987; 5: 269-79.

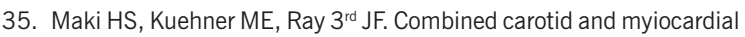
revascularisation. Am J Surg 1989; 158: 443-5.

36. Daily PO, Freeman RK, Dembitsky WP et al. Cost reduction by combined carotid endarterectomy and coronary artery bypass grafting. J Thorac Cardiovasc Surg 1996; 111: 1185-93.

37. Schwartz RL, Garrett JR, Karp RB et al. Simultaneous myocardial revascularization and carotid endarterectomy. Circulation 1982; 66: 97-101.

38. Slogoff S, Girgis KZ, Keats AS. Etiologic factors in neuropsychiatric complications associated with cardiopulmonary bypass. Anesth Analg 1982; 61: 903-11.

39. Hertzer NR, McCarthy RJ, Najafi H et al. Staged and combined surgical approach to simultaneous carotid and coronary vascular disease. Surgery 1978; 84: 803-11.

40. Mehigan JT, Buch WS, Pipkin RD et al. A planned approach coexistent cerebrovascular disease in coronary artery bypasss candidates. Arch Surg 1997; 112: 1403-9.

41. Jones EL, Craver JM, Michalik RA et al. Combined carotid and coronary operations: when are they necessary? J Thorac Cardiovasc Surg 1984; 87: 7-16.

42. Perler BA, Burdick JF, Minken SL, Williams GM. Should we perform carotid endarterectomy synchronously with cardiac surgical procedures. J Vasc Surg 1988; 8: 402-9.

43. Hertzer NR, Loop FD, Taylor PC, Beven EG. Combined myocardial revascularisation and carotid endarterectomy: operative and late results in 331 patients. J Thor Cardiovasc Surg 1983; 85: 577-89.

44. Bass A, Krupski WC, Dilley RB et al. Combined carotid endarterectomy and coronary artery revascularization: a sobering review. Isr J Med Sci 1992; 28:27-32

45. Bonamigo TP, Lucchese FA. Cirurgia simultânea das artérias carótida e coronária. IN Doenças da aorta e seus ramos. Bonamigo-BurihanCinelli-Von Ristow. São Paulo: Fundo Editorial BYK, 1991, 163-9.

46. Sundt TM, Sandok BA, Whisnant JP. Carotid endarterectomy: complications and preoperative assessment of risk. Mayo Clin Proc 1975; 50: 301

47. McKhann GM, Goldsborough MA, Borowicz LM et al. Predictors of stroke risk in coronary artery bypass patients. Ann Thorac Surg 1997; 63: 516-21.

48. John R, Choudhri AF, Weinberg AD et al. Multicenter review of preoperative risk factors for stroke after coronary artery bypass grafting. Ann Thorac Surg 2000; 69: 30-6. 
49. Barnes RW. Asymptomatic carotid disease in patients undergoing major cardiovascular operations: can prophylatic endarterectomy be justified? Ann Thorac Surg 1986; 42: 536-40.

50. Brown KR, Kresowik TF, Chin MH et al. Multistate ppulation-based outcomes of combined carotid endarterectomy and coronary artery bypass. J Vasc Surg 2003; 37: 32-9.

51. Naylor R, Cuffe RL, Rothwell PM, Loftus IM, Bell PRF. A systematic review of outcome following synchonous carotid endarterectomy and coronary artery bypass: influence of surgical and patient variables. Eur J Vasc Endovasc Surg 2003; 26: 230-41.
52. Fichino MZS, Paulista PP, Souza LCB et al. Endarterectomia e revascularização simultânea do miocárdio. Arq Bras Cardiol 1983; 41(4): 323-6.

53. Souza JM, Berlinck MF, Oliveira PAF et al. Cirurgia de revascularização do miocárdio associada a endarterectomia de carótida. Rev Bras Cir Cardiovasc 1995, 10(1): 43-9.

54. Takach TJ, Reul GJ, Cooley DA et al. Is an integrated approach warranted for concomitant carotid and coronary artery disease? Ann Thorac Surg 1997; 64: 16-22. 